\title{
Mesangiogenic progenitor cells are forced toward the angiogenic fate, in multiple myeloma
}

\author{
Simone Pacini ${ }^{1}$, Marina Montali ${ }^{1}$, Francesco Mazziotta ${ }^{2}$, Claudia P. Schifone ${ }^{1}$, Lucia \\ Macchia $^{3}$, Vittoria Carnicelli ${ }^{4}$, Francesca M. Panvini ${ }^{5}$, Serena Barachini ${ }^{1}$, Laura No \\ tarfranchi ${ }^{6}$, Giovanni Battista Previti ${ }^{7}$, Gabriele Buda ${ }^{1}$ and Mario Petrini ${ }^{1}$ \\ ${ }^{1}$ Department of Clinical and Experimental Medicine, Hematology Division, University of Pisa, Pisa, Italy \\ ${ }^{2}$ GeNOMEC School of Doctorate, University of Siena, Siena, Italy \\ ${ }^{3}$ Department of Laboratory Medicine, Azienda Ospedaliero-Universitaria Pisana, Pisa, Italy \\ ${ }^{4}$ Department of Surgical, Medical, and Molecular Pathology and Critical Care Medicine, University of Pisa, Pisa, Italy \\ ${ }^{5}$ Institute of Life Sciences, Sant'Anna School of Advanced Studies, Pisa, Italy \\ ${ }^{6}$ Department of Medicine and Surgery, Hematology Division, University of Parma, Parma, Italy \\ ${ }^{7}$ Unit of Anesthesia and Intensive Care, University of Sassari, Sassari, Italy \\ Correspondence to: Simone Pacini, email: simone.pacini@do.unipi.it \\ Keywords: multiple myeloma; bone marrow microenvironment; angiogenesis; osteogenesis; mesangiogenic progenitor cells \\ Received: July 18, $2019 \quad$ Accepted: October 04, $2019 \quad$ Published: November 26, 2019 \\ Copyright: Pacini et al. This is an open-access article distributed under the terms of the Creative Commons Attribution License 3.0 \\ (CC BY 3.0), which permits unrestricted use, distribution, and reproduction in any medium, provided the original author and source \\ are credited.
}

\section{ABSTRACT}

Multiple myeloma (MM) progresses mainly in the bone marrow where the involvement of a specific microenvironment plays a critical role in maintaining plasma cell growth, spread, and survival. In active disease, the switch from a pre-vascular/ non-active phase to a vascular phase is coupled with the impairment of bone turnover. Previously, we have isolated Mesangiogenic Progenitor Cells (MPCs), a bone marrow population that showed mesengenic and angiogenic potential, both in vitro and in vivo. MPC differentiation into musculoskeletal tissue and their ability of sprouting angiogenesis are mutually exclusive, suggesting a role in the imbalancing of the microenvironment in multiple myeloma.

MPCs from 32 bone marrow samples of multiple myeloma and 23 nonhematological patients were compared in terms of frequency, phenotype, mesengenic/ angiogenic potential, and gene expression profile. Defective osteogenesis was recorded for MM-derived MPCs that showed longer angiogenic sprouting distances respect to non-hematological MPCs, retaining this capability after mesengenic induction. This altered MPCs differentiation potential was not detected in asymptomatic myelomatous disease.

These in vitro experiments are suggestive of a forced angiogenic fate in MPCs isolated from MM patients, which also showed increased sprouting activity. Taking together our results suggest a possible role of these cells in the "angiogenic switch" in the MM micro-environment.

\section{INTRODUCTION}

Tumor microenvironment contributes to disease progression in most haematological malignancies [1]. In particular, in Multiple Myeloma (MM) the interactions between malignant plasma cells (PCs) and the bone marrow (BM) niche sustain and promote tumor growth [2]. Endothelial cells, stromal cells, osteoblasts, osteoclasts, and immune cells together with the extracellular matrix are involved in the process. Cross-talks between neoplastic 
cells and BM create a suitable microenvironment for disease development and are responsible for the hallmarks of MM progression: osteolysis and angiogenesis [3].

MM cells develop complex interactions with BMmesenchymal stromal cells (BM-MSCs) supporting tumor survival and chemoresistance [4]. Moreover, BMMSCs appear to have reduced osteogenic potential and to stimulate proliferation/activity of osteoclasts, thus contributing to osteolysis [5]. Osteoclasts also establish a feed-forward relationship with PCs, which further supports tumor growth [6]. The role of osteoblasts and osteocytes is yet to be clarified although some authors showed MM cells to inhibit osteoblast proliferation, differentiation, and activity through different molecular pathways while promoting osteocyte apoptosis [6].

In BM microenvironment the imbalance between pro- and anti-angiogenic factors represents a key step for tumor progression [7]. To date, the underlying pathophysiology of the pro-angiogenic switch in MM is only partially understood. The release of pro-angiogenic molecules by BM cells has a non-negligible role in the loss of angiostasis in Monoclonal Gammopathy of Undetermined Significance (MGUS) pre-tumoral quiescent condition [7]. Furthermore, interactions between malignant cells and BM microenvironment have been shown to enhance angiogenesis and to promote tumor progression in a vicious circle [8]. MM progression appears to be accompanied by an increase in angiogenesis from the MGUS pre-vascular/non-active phase to the vascular phase characterizing active MM [8]; [7].

Since first identification, MSCs have been associated to bone turnover and metabolism in a number of pathological conditions [9], including MM [5]. The secretion of cytokines and stimulating factors points to MSC involvement in vascular growth [10] although MSC ability to differentiate directly into endothelial cells is still debated. In vitro studies showed contradictory results possibly due to the heterogeneity of bulk cultures [11]. Indeed, culture conditions can select diverse sub-populations of MSC progenitors with specific differentiation potentials, as shown by the effect of human sera on the isolation of Mesangiogenic Progenitor Cells (MPCs) [12].

MPCs have been identified in human BM under specific culture condition. Cells are round shaped with a refractive central core and fringed periphery, and they show trypsin-resistant plastic adherence [13]. MPCs express pluripotency-associated genes [14] and have been characterized as resting cells, retaining both mesengenic and angiogenic potential [15]. In particular, MPC mesengenic differentiation is a two step process. First, activation of non-canonical Wnt-5/calmodulin pathway drives cells to the P1-MSC stage. Addition of calmodulin antagonist calmidazolium chloride (CLMDZ) [16], during this step, results in the complete ablation of the mesengenic differentiation while have no effect on resting MPCs or their endothelial differentiation [17]. These data not only confirmed the activation of calmodulin during the induction of MPCs into P1-MSCs, but also correlate the sensibility to CLMDZ with this specific step of mesengenic differentiation. In fact, the second passage under mesengenic stimulation, leading P1-MSC into P2MSC showing standard MSC morphology, phenotype and function, is not affected by CLMDZ treatment and apparently involving the canonical Wnt pathway [17]. MPCs can undergo the angiogenic fate under VEGF stimulus by two step culture, with angiogenesis prompted by MPC 3D-spheroids let sprouting in extracellular matrix protein gel. MPC angiogenic potential is lost after mesengenic induction, confirming the two fates to be mutually exclusive [15]. As stated above, the MPC endothelial differentiation is not impaired by the addition of CLMDZ, however a specific inhibitor of the MPC angiogenic fate has not been tested before. In 2016, a specific BM cell population has been identified by multicolor flow cytometry as the putative and unique MPC in vivo progenitor [18]. Back-gating lineage markers on CD18 vs CD31 scatter plots allowed identification of seven clusters associated to most of the BM mononuclear cell populations. An eighth population (Pop\#8) has been also identified and characterized as CD $45^{\mathrm{dim}} \mathrm{CD} 33^{+} \mathrm{CD} 11$ $\mathrm{b}^{\text {neg }} \mathrm{CD} 64^{\text {bright }} \mathrm{CD} 31^{\text {bright }} \mathrm{CD} 14^{\text {neg }}$, resembling the phenotype of immature monocyte precursors. Interestingly, sorting experiment demonstrated that this latest population is the unique BM population able to generate MPCs in culture, identifying Pop\#8 as the ex vivo ancestor of those mesangiogenic cells [18].

From the first evidence of their mesangiogenic potential, we hypothesize a role for MPCs in BM stroma re-modeling and homeostasis. However, a definitive demonstration of the involvement of MPCs in the maintaining the bone marrow microenvironment is still lacking. Nonetheless, due to their differentiation potential, it is reasonable hypothesize that the MPC behavior could be altered during MM development and progression as a consequence of the deregulation that malignant PCs exert on BM stroma. Here we investigated possible alteration of the MPC in vitro properties, analyzing MPC frequency and characterizing their mesangiogenic potential in $\mathrm{MM}$ patients and compared to non-haematological $(\mathrm{NH})$ subjects. We also tested the effect of bortezomib (BTZM), the first pretoasome inhibitor applied in the treatment of MM patients with potent anti-angiogenic activity in bone marrow [19], on MPC angiogenic fate.

\section{RESULTS}

\section{PC bone marrow infiltration reduces the percentage of Pop\#8 sub-population, in MM patients.}

The percentage of Pop\#8 sub-population was significantly lower $(p<0.001)$ in $\mathrm{BM}$ from MM patients $(0.26 \pm 0.06 \%, \mathrm{n}=21)$ as compared to $\mathrm{NH}$ patients $(1.56$ 
$\pm 0.12 \%, \mathrm{n}=20)$ (Figure $1 \mathrm{~A}, 1 \mathrm{~B})$. Such reduced frequency could be the consequence of malignant $\mathrm{PC}$ infiltration in MM patients that has been shown to reach up to $70 \%$ of $\mathrm{BM}$, resulting in hypoplasia of the main haematopoietic lineages. Indeed, absolute count of Pop\#8 cells resulted not significantly different $(52.5 \pm 8.6$ cells $/ \mu l, n=20$ in $\mathrm{NH} v s 41.6 \pm 10.2$ cells/ $\mu \mathrm{l}, \mathrm{n}=21$ in $\mathrm{MM}, p=0.424)$ and its percentage negatively correlated with the percentage of CD138 ${ }^{\text {bright }} \mathrm{CD} 45^{\mathrm{dim} / \mathrm{neg}}$ (Spearman $R=-0.569, p<0.05$ ) that ranged from $0.80 \%$ to $38.60 \%$ of total BM cells, in MM samples (Figure 1C). Moreover, positive correlation (Spearman $R=0.827, p<000.1$ ) between Pop\#8 and CD34 ${ }^{+}$ cell frequency was detected, confirming that reduction in Pop\#8 sub-population was coupled with reduced haematopoiesis (Figure 1D). Similar results were obtained for the mature monocyte population $(74.3 \pm 13.9$ cells $/ \mu 1$, $\mathrm{n}=20$ in $\mathrm{NH}$ vs $77.3 \pm 14.7$ cells $/ \mu \mathrm{l}, \mathrm{n}=21$ in $\mathrm{MM}, p=0.890$ )

\section{MPCs from MM patients are forced toward the angiogenic fate, under mesengenic stimuli}

After a week of culture under MPC selective conditions, three samples of the $\mathrm{NH}$ and two of the MM group were excluded from the study due to a low yield/ purity of the recovered cell population. In the remaining samples, the mean purity of MPC cultures resulted higher than $90 \%$ both for $\mathrm{NH}(96.7 \pm 1.5 \%, \mathrm{n}=20)$ and $\mathrm{MM}$ samples $(94.3 \pm 2.6 \%, n=21)$ with undetectable CD14 ${ }^{\text {bright }}$ population, as expected applying selective culture [20]. In accordance to the observed reduction of Pop\#8 in MM patients, MPC frequency from primary cultures was found to be significantly $(p<0.001)$ lower in MM samples $(0.57 \pm$ $0.09 \%, \mathrm{n}=21)$ as compared to NH samples $(1.17 \pm 0.17 \%$, $\mathrm{n}=20$ ) (Figure 2A).

During the in vitro mesengenic differentiation toward P2-MSCs, no difference was found between MM and $\mathrm{NH}$ patients, indicating MPC mesengenic potential to apparently remain unaffected. Also, similar growth curves were recorded (Figure 2B). However, terminal osteogenic differentiation resulted impaired in P2-MSCs derived from MM patients. The significant $(p<0.01)$ reduction of mineralized area in MM samples $(2.88$ $\pm 0.38 \%, \mathrm{n}=21)$ as compared to $\mathrm{NH}(11.73 \pm 1.91 \%$, $\mathrm{n}=20)$ casted doubts on the genuineness of mesengenic MPC differentiation into MSC-like cells (Figure 2C). The hypothesis was confirmed by the calmodulin
A

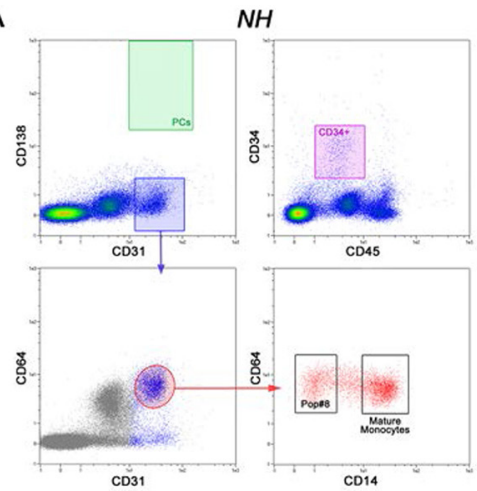

B

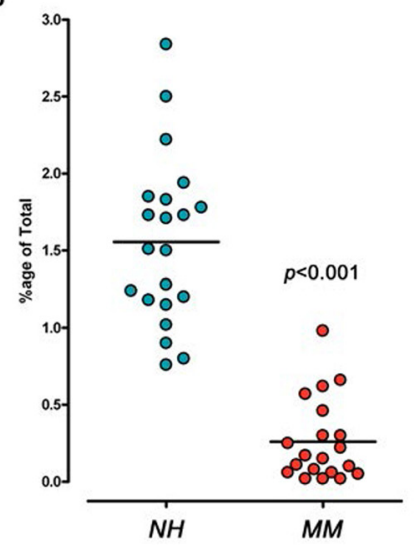

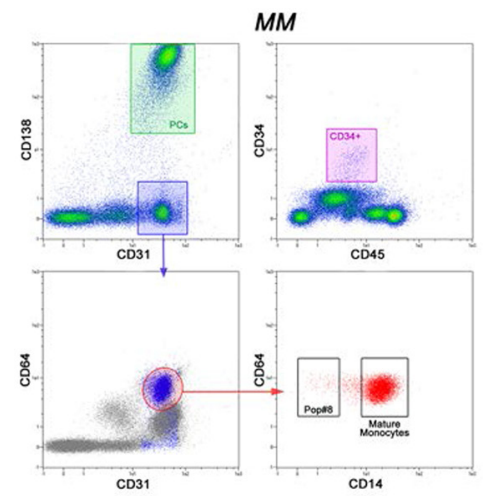

C
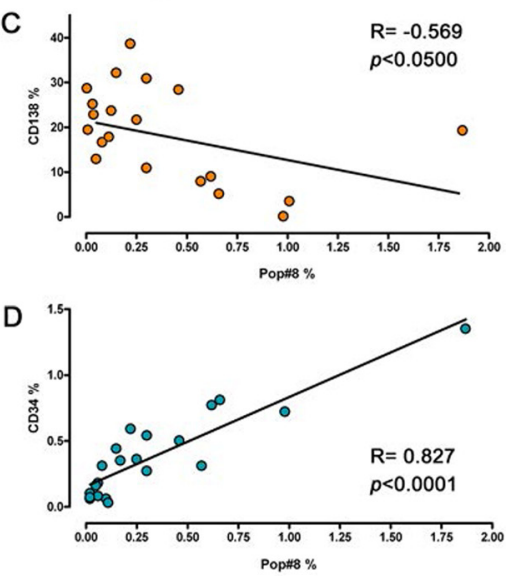

Figure 1: Frequency of Pop\#8 sub-population in MM patients. (A) MPCs and haematopoietic progenitors were identified by flow cytometry as $\mathrm{CD} 31^{\text {bright }} \mathrm{CD} 64^{\text {bright }} \mathrm{CD} 14^{\text {neg }}$ (black box) and $\mathrm{CD} 34^{+} \mathrm{CD} 45^{\text {dim }}$ (purple box), respectively. Malignant PCs were identified as CD138 $8^{\text {bright }}$ CD45 ${ }^{\mathrm{dim}}$ (green box). (B) Frequency of Pop\#8 was significantly lower in MM patients as compared to NH patients. (C) Pop\#8 frequency negatively correlated with the percentage of PCs and (D) was associated to reduced haematopoiesis. 
signalling pathway inhibition induced by CLMDZ during MPC differentiation into P1-MSCs. Addition of $0.5 \mu \mathrm{M}$ CLMDZ resulted in about $35 \%$ inhibition of the AlamarBlue reduction in NH samples $(35.05 \pm 5.11 \%)$ as compared to untreated cultures $(54.09 \pm 3.89 \%)(n=12$; $p<0.01)$. Conversely, MPC mesengenic differentiation in MM samples was unaffected by CLMDZ treatment (43.31 $\pm 2.91 \%$ vs $47.70 \pm 3.10 \%$ of untreated cultures, $\mathrm{n}=12$ ), supporting the hypothesis that the recorded proliferation could not be associated to genuine mesengenic differentiation (Figure 2D). Treatment with 2 nM BTZM significantly $(p<0.01)$ promoted MPC mesengenic differentiation in $\mathrm{NH}$ samples $(60.63 \pm 2.91 \%$ vs 47.93 $\pm 4.16 \%$ of untreated cultures, $n=16)$, whereas $3 \mathrm{nM}$ BTZM had no effect $(40.73 \pm 3.44 \%, n=16)$, showing that BTZM posses a pro-osteogenic and an anti-angiogenic activity on normal MPC similarly to what reported on other bone marrow stromal cells [19], [21]. Conversely, BTZM impaired the proliferation of MPCs from MM patients during differentiation into P1-MSCs, in a dosedependent manner $(41.19 \pm 2.82 \%, p<0.01$, at $2 \mathrm{nM}$ and $31.62 \pm 2.99 \%, p<0.001$, at $3 \mathrm{nM}$ vs $50.63 \pm 2.91 \%$ of untreated cultures, $n=13$ ) (Figure 2E), suggesting that the differentiation occurred could not be mesengenic but angiogenic.

MM patient-derived MPCs showed increased angiogenic potential with significantly $(p<0.05)$ longer sprouting distances $(442.2 \pm 49.5 \mu \mathrm{m}, \mathrm{n}=16)$ as compared to $\mathrm{NH}$ patients $(288.8 \pm 58.3 \mu \mathrm{m}, \mathrm{n}=10)$. BTZM impaired sprouting angiogenesis in a dose dependent-manner, both in NH and MM patient-derived MPCs, showing similar inhibition curve slopes (Figure 2F).

Assessement of angiogenic potential after MPC mesengenic induction revealed significant differences between "active disease" (MM) and SMM patients $(p<0.001)$ as well as between MM and NH patients $(p<0.01)$. P1-MSCs from MM patients showed moderate reproducible sprouting activity with a front of invading cells at around $200 \mu \mathrm{m}$ from the spheroid edge (197.1 \pm $22.9 \mu \mathrm{m}, \mathrm{n}=10$ ) at a difference with P1-MSCs from $\mathrm{NH}$ and SMM patients that revealed branching of cellular protrusions only within $100 \mu \mathrm{m}$ from the spheroid edge $(96.5 \pm 22.9 \mu \mathrm{m}, \mathrm{n}=6$ and $57.8 \pm 14.67 \mu \mathrm{m}, \mathrm{n}=8$, respectively) (Figure 3A). This data further support the idea that P1-MSCs from MM patients were not mesenchymal cells but early angiogenic cells, as suggested by the inhibition experiments with CLMDZ and BTZM. The residual angiogenic potential of MM P1-MSCs was lost after the second step of mesengenic differentiation, as shown by lack of sprouting activity in P2-MSC derived spheroids, similarly to previous results in orthopedic patients [15]. Taking together our results strongly support a forced angiogenic fate for MPCs from MM patients, during the differentiation into P1-MSCs.

\section{Gene expression profiling of P1-MSCs supports the hypothesis of forced MPC angiogenic fate}

Unsupervised gene expression clustering analysis of 86 target genes revealed two main clusters associated
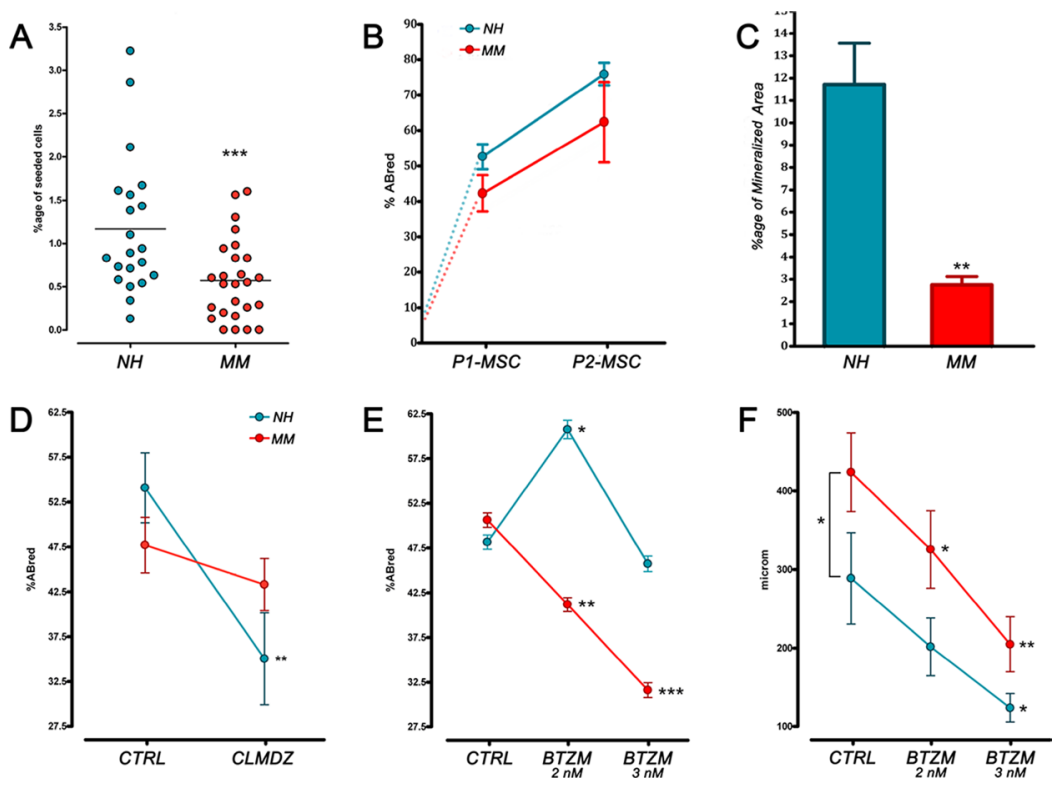

Figure 2: MPC frequency and differentiative potential in NH and MM patient. (A) MPC frequency from primary cultures was found to be significantly lower in MM samples. (B) Under mesengenic stimuli no difference was found between MM and NH patientderived MPCs, neither in growth curves. (C) After 21 days of terminal osteogenic differentiation, a reduced mineralized area was detected in MM patients indicating defective calcium deposition. (D, E) BTZM inhibited MM patient-derived MPC differentiation into P1-MSC at a difference with CLMDZ. (F) Under angiogenic stimuli sprouting from MPC 3D spheroids resulted in longer sprouting distances in MM samples. BTZM impaired sprouting in a dose-dependent manner. $\left({ }^{*} p<0.05,{ }^{* *} p<0.01,{ }^{* * *} p<0.001\right)$ 

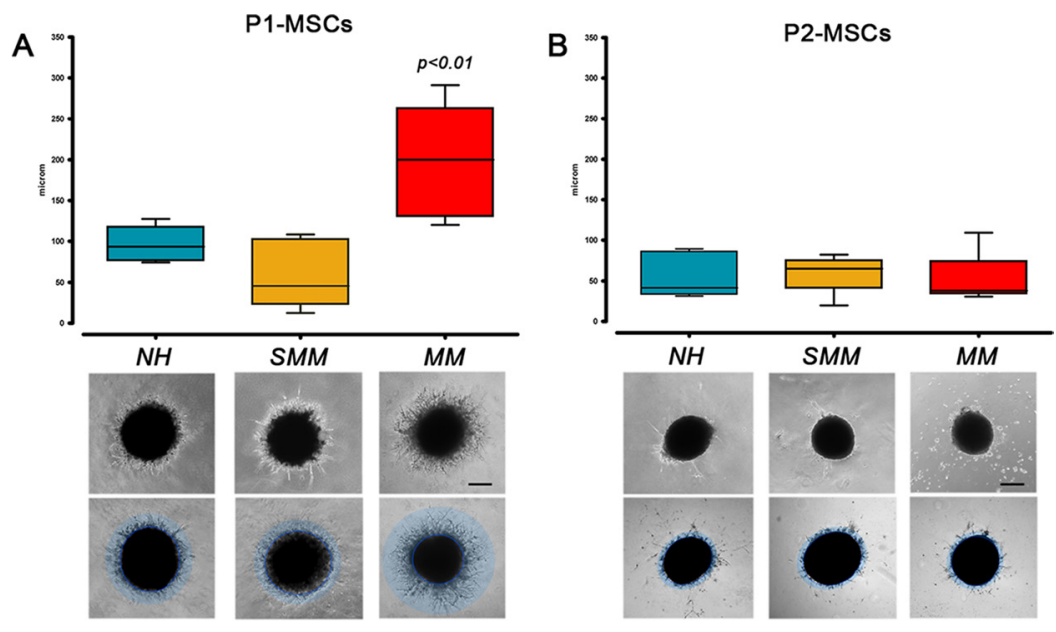

Figure 3: Angiogenic potential after mesengenic induction. (A) P1-MSCs from MM patients retained higher sprouting activity under angiogenic stimuli with respect to P1-MSCs from SMM and NH patients. (B) This residual angiogenic potential was lost after the final step of mesengenic differentiation to P2-MSCs. $($ scale bar $=200 \mu \mathrm{m})$

to the MPC (pale blue box) or MSC (pink box) phenotype (Figure 4A). After mesengenic induction, P1-MSC samples from $\mathrm{NH}$ and $\mathrm{MM}$ patients both showed increased expression of MSC-related genes and reduced expression of MPC-related genes. Single gene analysis revealed lower expression of the most specific previously described MPC markers, including osteopontin (SPP1), CD18 (ITGB2), CD11b (ITGAM), CD11c (ITGAX), and matrix metallopeptidase 9 (MMP9), from 0.07 to 0.03 fold, in MM samples ( $\mathrm{n}=5$, Figure 4B). The down-regulation of MPC-associated genes is expected during differentiation into P1-MSCs, however in MM samples silencing of these genes resulted about $2 \operatorname{logs}$ more consistent respect to NH (SPP1: $-5.81 \times 10^{2} v s-4.00 \times 10^{1} ;$ ITGB2: -1.10 $\times 10^{3}$ vs $-7.68 \times 10^{1}$; ITGAM: $-1.50 \times 10^{3}$ vs $-8.77 \times 10^{1}$; ITGAX: $-1.50 \times 10^{3} v s-6.5 \times 10^{1} ; M M P 9$ : $-4.54 \times 10^{3} v s-1.13 \times 10^{2}$, $p<0.05, \mathrm{n}=5$ ). Conversely, a seven-fold higher expression of Dickkopf WNT signaling pathway inhibitor 1 (DKK1, 6.96 $\pm 2.87, \mathrm{n}=5, p<0.05)$ and endomucin $(E M C N, 7.28 \pm 3.59$, $\mathrm{n}=5, p<0.05$ ) has been detected in P1-MSCs derived from MM samples. These data suggest that, in MM patients, the unexpected angiogenic potential at P1-MSCs is not correlated to the maintaining of the MPC undifferentiated condition. Moreover, the up-regulation of $D K K 1$ could be suggestive for a hampered mesengenic fate, associated to the activation of canonical Wnt $/ \beta$-catenin signaling.

\section{DISCUSSION}

In recent years impairment of bone turnover and angiogenesis have been described as two hallmarks of tumor microenvironment in MM [22]; [23]. The microenvironment has a fundamental role in supporting MM, since malignant PCs survive almost exclusively in BM [3]. Indeed, novel treatment strategies target malignant tumor cells as well as their microenvironment, allowing deep responses and fuelling the debate cure $v s$ long-term response [24]. In the present study, we showed altered in vitro response to differentiation stimuli of MPCs derived from MM patients, suggesting that these cells could play a role in: i) tumor growth, contributing to new vessels formation and ii) osteolysis, reducing the osteblastogenesis.

It is known that BM infiltration by myeloma cells reduce physiological hematopoiesis due to the invasion of BM cavity [25]. Consistently, in our study BM resident MPC precursor Pop\#8 cells [18] and classical monocytes, although were found at lower frequencies as a consequence of tumor burden, resulted unvaried in their absolute count in samples from MM patients as compared to $\mathrm{NH}$ patients.

As regard to MPC differentiative potential, we found apparently no significant difference in mesengenic differentiation to P2-MSCs between NH and MM patients. On the other hand, osteogenic terminal differentiation was significantly compromised in patients diagnosed with MM. A similar behavior had been related to inhibition of the noncanonical Wnt5a/Ror2 pathway during MSC differentiation to osteoblasts in MM [26]. In our experimental setting data suggest a switch, possibly induced by malignant PCs, from genuine mesengenic differentiation leading to MSC-like cells in favor of a different MPC fate. BTZM treatment inhibited MM patient-derived MPC differentiation into P1-MSC at a difference with CLMDZ, supporting the idea of MPC fate restriction notwithstanding the observed defective osteogenic terminal differentiation. We previously demonstrated Wnt5a non-canonical pathway involvement in MPC differentiation to P1-MSCs [17] and also evidenced the presence of undifferentiated MPCs within MSC bulk cultures [11]. Therefore, the osteogenic differentiation impairment described by Bolzoni et al. [26] would be a consequence of MSC bulk culture heterogeneity. In our scenario, CLMDZ treatment could act on MPCs rather than MM-derived MSCs re-balancing their differentiative potential toward 

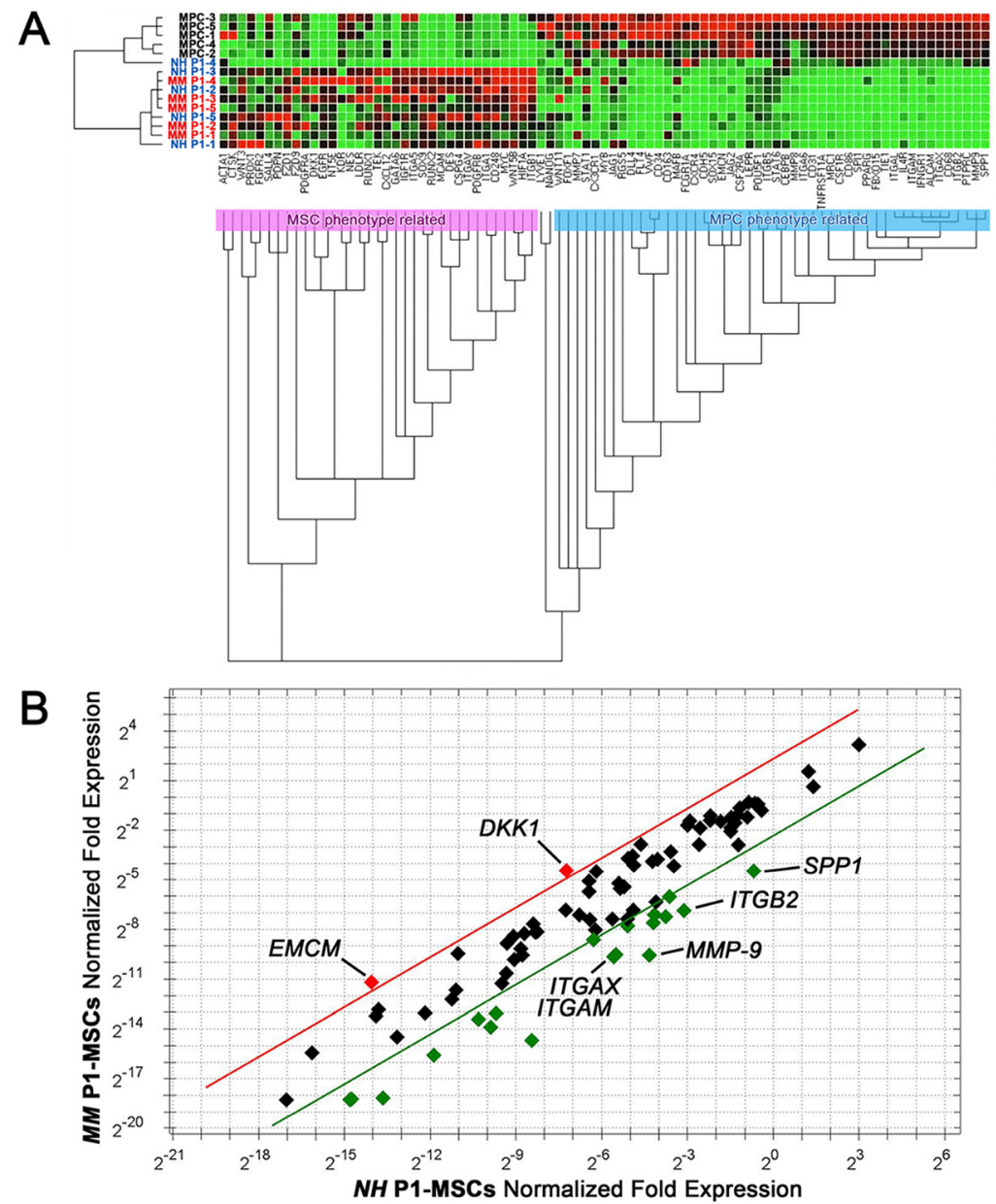

Figure 4: Gene expression profile of P1-MSCs from NH and MM patients. (A) Clustering gene expression analysis of samples from NH (blue font) and MM (red font) patients showed up-regulation of MSC-related genes (red dots) and down-regulation of MPCrelated genes (green dots). (B) Single gene expression analysis revealed increased expression of DKK1 and EMCN in MM samples (red dots). Specific MPC markers showed increased expression in NH samples (green dots). Five-fold ratio of normalized fold expression was fixed as a threshold (red and green lines).

mesengenesis. On a larger scale one could envisage the inhibition of musculoskeletal fate in MM patients to be localized at the stage of MPCs rather than MSCs.

MM patient-derived MPCs showed increased angiogenic potential as compared to NH patients. BTZM impaired sprouting angiogenesis in a dose dependentmanner, both in NH and MM MPCs. Assessement of angiogenic potential after MPC mesengenic induction revealed significant differences between "active disease" (MM) and SMM patients as well as between MM and NH patients. P1-MSCs from MM patients still showed moderate reproducible sprouting activity proving a persistent angiogenic potential. This latest was lost after the second step of mesengenic differentiation, similarly to previous results in orthopedic patients [15] except for the defective osteogenic terminal differentiation evidenced by the inefficient calcium deposition. Our set of data is indicative of progressive loss of MPC angiogenic potential without restoration of their in vitro osteogenic activity once abandoned the in vivo conditioning myelomatous BM microenvironment. The forced angiogenic fate of MPCs appears to be related to MM active stage, since sprouting activity in SMM patients was comparable to $\mathrm{NH}$ patients.

Gene expression analysis of a set of genes involved in MPC mesenchymal differentiation revealed overlapping profiles between NH and MM derived P1-MSCs. The only exception was represented by a consistent up-regulation of endomucin $(E M C N)$, recently described as a marker for type $\mathrm{H}$ vessels. This sub-set of BM microvessels has been proposed to mediate local growth of vasculature and their localization at the distal end of the arterioles suggest for them to support a specialized metabolically active microenvironment, both in mice and humans [27]; [28]. Creating a privileged access to nutrients and oxygen, $\mathrm{CD} 31^{\text {hi }} \mathrm{Emcn}^{\text {hi }}$ capillaries may represent a key factor sustaining malignant BM cells. DKK1, whose downregulation has been detected during the late stage of MPC mesengenic differentiation into P2-MSCs [17], showed 
increased expression in MM derived P1-MSCs. Such up-regulation could prevent differentiation into genuine MSC-like cells and force angiogenic commitment.

Prior studies described BTZM effect on tumor microenvironment focusing on the induction of osteogenic terminal differentiation [21]; [29]. Our in vitro evidence provides a possible additional effect of BTZM treatment. Basing on the assumption that mesenchymal differentiation in MM is partly blocked at the stage of MPCs that are forced toward angiogenesis, we hypothesize that malignant PCs could modify the MPC fate in favor of tumor growth and consequently affect osteogenesis. BTZM anti-angiogenic effect would therefore be able to revert the MPC angiogenic switch restoring MPC mesengenic potential. We also believe that the unbalance in the angiogenic potential shown by MM vs SMM derived P1MSCs would localize MPC forced angiogenesis in vivo at the switch from asymptomatic SMM to active MM. In this scenario, BTZM activity on MPCs not only would be able to restore a balanced mesangiogenesis in MM, but also in SMM could act protecting from the angiogenic switch, at the basis of the disease progression. Even if further data supporting this hypothesis are needed, our results could therefore have relevant clinical implications to improve patients' outcome. Current recommendations continue to be SMM patient observation. New indications of therapy would contribute to prevent progression ad hopefully eradicate the disease at early stage [30]. The unaffected mesenchymal and osteoblastic differentiation we observed under low tumor burden supports the idea of treating SMM patients before substantial microenvironmental deregulation [31]. Moreover, in agreement with previous studies focused on the role of proteasome inhibitors for post-transplant maintenance [32]; [33]; [34]; [35], we believe that BTZM could establish a non-permissive tumor microenvironment by blocking the MPC angiogenic switch, thus deepening or maintaining disease remission.

In conclusion, our in vitro cell system appeared to be consistent with the natural history of MM, suggesting a similar MPC behavior in vivo. Our data allowed to show MM angiogenesis and osteolysis not be seen as independent aspects of the same disease, but rather as coupled processes responsible for tumor progression. We also evidenced the expression of a number of genes, including $E C M N$ and $D K K 1$, possibly involved in the deregulation of tumor microenvironment. Further studies will be required to evaluate whether targeting the MPC fate could interfere with tumor growth and restore osteogenesis, as well as hampering disease progression.

\section{METHODS}

\section{Bone marrow mononuclear cell isolation}

Human BM aspirates were collected by iliac crest puncture from 32 patients $(16 \mathrm{~F} / 16 \mathrm{M}$, median age 68 , range 52-85) after written consent (Supplementary Table 1). The experimental group included $23 \mathrm{MM}$ (7 ISS I, 11 ISS II, and 3 ISS III) patients (15 with bone lytic lesions) and 9 Smoldering Multiple Myeloma (SMM) patients, either newly diagnosed or free from therapies for almost two years [36]. No patients with solitary myeloma or extra medullary plasmocytoma were enrolled. BM aspirates were also collected from 23 control patients $(13 \mathrm{~F} / 10 \mathrm{M}$, median age 66, range 50-72) with non hematological disease $(\mathrm{NH})$. Samples were collected during hip replacement orthopedic surgery immediately after femoral neck osteotomy. The study was performed according to the declaration of Helsinki and sample collection protocol approved by the ethical committee of the Azienda Ospedaliero-Universitaria Pisana (committee approval number: 48812/07).

BM samples were diluted 1:4 in Dulbecco's modified phosphate-buffered saline (D-PBS; Thermo Fisher, Waltham, MA-USA) and gently layered on Ficoll-Paque $^{\circledR}$ PREMIUM (GE Healthcare, Uppsala, Sweden). Samples were centrifuged at $400 \mathrm{~g}$ for $25 \mathrm{~min}$ and BM mononuclear cells (BM-MNCs) harvested at the interface, washed twice in D-PBS and counted by Bürker hemocytometer.

\section{Flow cytometry}

Freshly isolated BM-MNCs were processed for multicolor flow cytometry analysis. Cells were incubated for $30 \mathrm{~min}$ at $4^{\circ} \mathrm{C}$ with fluorochrome-conjugated antibodies: anti-CD34 VioBlue ${ }^{\circledR}$, anti-CD64 FITC, antiCD138 PE, anti-CD14 PerCP-Cy5.5, anti-CD31 PE-Cy7, anti-CD56 APC, and anti-CD45 APC-Vio770 ${ }^{\circledR}$ (Miltenyi Biotec, Bergisch Gladbach, Germany). After washing in MACSQuant ${ }^{\circledR}$ Running Buffer (Miltenyi Biotec), cells were sorted by MACSQuant ${ }^{\circledR}$ Analyzer (Miltenyi Biotec) equipped with MACSQuantify ${ }^{\circledR}$ analysis software (Miltenyi Biotec). After exclusion of debris on SSC vs FSC plot and doublet on FSC-H vs FSC-A, events were plotted on CD138 vs CD31 density plot. Two different gates were identified to select $\mathrm{CD} 138^{\text {bright }} \mathrm{CD} 31^{+}$and CD138 ${ }^{\text {neg }} \mathrm{CD} 31^{\text {bright }}$. CD138 $8^{\text {bright }} \mathrm{CD} 31^{+}$were then plotted on CD45 vs CD138 and CD138 bright CD $31^{+}$CD $45^{\text {dim/neg }}$ events recorded as PCs. As previously reported, two further subgates of the CD138 ${ }^{\text {neg }} \mathrm{CD} 31^{\text {bright }}$ population were defined for accurate quantification of Pop\#8 sub-population as CD3 $1^{\text {bright }} \mathrm{CD} 64^{\text {bright }} \mathrm{CD} 14^{\text {neg }}[18]$ and mature monocytes as CD31 $1^{\text {bright }} \mathrm{CD} 64^{\text {bright }} \mathrm{CD} 14^{\text {bright }}, \mathrm{CD} 45^{\text {dim }} \mathrm{CD} 34^{+}$cells were also quantified (Figure 1A). Frequencies of all those cell sub-populations were calculated on singlet cellular events obtained excluding debris on SSC vs FSC and doublets on FSC-A vs FSC-H plots. Non parametric Wilcoxon's test for matched pairs and Spearman correlation test were performed by GraphPad Prism ${ }^{\circledR}$ software (GraphPad Software, San Diego, USA-CA). Absolute count of cells per $\mu \mathrm{l}$ was also recorded and the mean values were compared applying $t$-test. 


\section{BM-MNC culture under MPC selective conditions}

MPCs were isolated from BM-MNC cultures as previously described [20]. Briefly, cells were seeded from $6 \times 10^{5} / \mathrm{cm}^{2}$ to $8 \times 10^{5} / \mathrm{cm}^{2}$ on no gas-treated hydrophobic plastic flasks (GreinerBio-One, Kremsmünster, Austria) and cultured in Dulbecco's modified Eagle's medium (DMEM, Thermo Fisher) supplemented with $10 \%$ pooled human AB-type serum (PhABS, SeraLab, West Sussex, UK). Serum lots had been screened to provide optimal MPC isolation. Media were changed every $48 \mathrm{~h}$, and cultures maintained at $37^{\circ} \mathrm{C}$ in $5 \% \mathrm{CO}_{2}$, for 6-7 days. Cells were then detached by TrypLE Select ${ }^{\circledR}$ (Thermo Fisher) digestion and counted in Bürker hemocytometer. Flow cytometry was also applied to validate the MPC preparations. Around 100'000 cells from freshly detached MPC were stained with anti-CD45 APC-Vio770 ${ }^{\circledR}$, antiCD31 PE-Cy7, anti-CD18 FITC, anti-CD73 PE and anti-CD90 APC (Miltenyi Biotec). Cultures with MPC population, defined as $\mathrm{CD} 31^{+} \mathrm{CD} 18^{+} \mathrm{CD} 45^{\text {low }} \mathrm{CD} 73^{\text {neg }} \mathrm{CD} 9$ $0^{\text {neg }}$, lower than $90 \%$ were excluded from the study.

MPC frequency was calculated as ratio between MPCs obtained at the end of culture and total number of seeded cells. Statistical significance was assessed by Wilcoxon's test.

\section{Assessment of MPC differentiative potential}

MPC mesengenic, osteogenic, and angiogenic differentiation pathways were induced using previously described protocols [20].

\section{Mesengenesis}

Freshly detached MPCs obtained as above were seeded at $2 \times 10^{4}$ cells $/ \mathrm{cm}^{2}$ in TC-treated T25 flasks and let to adhere in DMEM/10\% PhABS. After $24 \mathrm{~h}$ medium was replaced with StemMACS ${ }^{\mathrm{TM}}$ MSC Expansion Media XF (Miltenyi Biotec) and cultures grown to confluence to obtain passage one MSCs (P1-MSCs). Cells were then detached with TrypLE Select ${ }^{\circledR}$ and sub-cultured 1:2 to confluence to obtain passage two MSCs (P2-MSCs). In parallel, 6-well plates were set up in duplicate to draw corresponding growth curves. AlamarBlue reduction assay was performed at day 7 and day 14 adding $10 \%$ PrestoBlue ${ }^{\circledR}$ Cell viability assay (Thermo Fisher) to culture medium. After $8 \mathrm{~h}$ incubation, $100 \mu \mathrm{l}$ of culture medium were harvested and absorbance was measured at 570 and $600 \mathrm{~nm}$ by Benchmark Plus microplate spectrophotometer (BioRad, Hercules, USA-CA). Percentage of reduced AlamarBlue was calculated according to manufacturer and two-tailed unpaired $t$-test was performed. Growth curves were also acquired after cell treatment with $0.5 \mu \mathrm{M}$ calmidazolium chloride (CLMDZ) (Sigma Aldrich, St. Louise, USA-MO) or 2 and $3 \mathrm{nM}$ bortezomib (BTZM) (Selleckchem, Houston, USA-TX).

\section{Osteogenesis}

P2-MSCs were detached, re-plated at 20.000 cells/ $\mathrm{cm}^{2}$ in 24-well TC-treated plates, and grown to confluence in MSC expansion medium. To induce osteogenesis, medium was replaced by StemMACS ${ }^{\text {TM }}$ OsteoDiff Medium (Miltenyi Biotec) and cultures maintained for 21 days (medium changed weekly). Control plates were set up in MSC expansion medium. Calcium deposits were revealed by OsteoImage ${ }^{\mathrm{TM}}$ Mineralization assay kit (Lonza, Basel, Switzerland) according to manufacturer. Pictures were taken using an inverted fluorescence DM IRB Leica microscope (Leica) equipped with LAS image acquisition software (Leica). Image analysis was performed by QWin image analysis software (Leica) to quantify the percentage of fluorescent (mineralized) areas. Two-tailed unpaired $t$-test was performed.

\section{Sprouting angiogenesis}

3D spheroids (6 to 8 ) were obtained by hanging drop method applying $1.5 \times 10^{5}$ cells/drop, as previously described [15]. After $24 \mathrm{~h}$, spheroids were gently laid on Geltrex $^{\circledR}$ (Thermo Fisher) thick gel and cultured for one week in EGM-2 medium (Lonza, Basel, Switzerland) with a single medium change. Sprouting angiogenesis was assayed on MPC derived spheroids, both in the presence ( 2 and $3 \mathrm{nM}$ ) or absence of BTZM, as well on P1- and P2-MSCs derived spheroids in the absence of BTZM. All samples were run in duplicate. Phase contrast pictures were taken at $100 \mathrm{X}$ magnification and sprouting distances quantified by QWin image analysis software (Leica) measuring the distance between the last invading cell and the spheroid edge. Measures were taken in blind by three separate researchers. Statistical analysis was performed by two-tailed unpaired $t$-test.

\section{Gene expression analysis of P1-MSCs}

Gene expression analysis was performed in P1MSCs, using NH-derived MPCs as undifferentiated control. Custom 96-well PrimePCR ${ }^{\text {TM }}$ Plates (BioRad, Hercules, USA-CA) containing primer sets for 86 target genes, 5 reference genes (Supplementary Table 2), and 5 internal controls were used for the gene expression profile assay. Total RNAs were purified from freshly detached cells by Direct-zol RNA MicroPrep Kit (Zymo Research, Irvine, USA-CA) and quantified with Qubit 4 Fluorometer (Thermo Scientific) by Qubit RNA HS Assay Kit (Termo Scientific). cDNAs were synthesized from $1 \mu \mathrm{g}$ total RNA using iScript gDNA Clear cDNA Synthesis Kit, according to manufacturer. qPCR was carried out by SsoAdvanced Unversal SybrGreen Supermix (BioRad) on iQ5 Real time PCR Detection System (BioRad), according to PrimePCR Array ${ }^{\mathrm{TM}}$ manufacturer. Fold change ${ }^{\Delta A} \mathrm{C}_{t}$ method calculations and statistical analysis were carried out using PrimePCR ${ }^{\text {TM }}$ Analysis software (BioRad). $\mathrm{C}_{\mathrm{t}}$ values over 35 were considered as "no expression". Following best 
housekeeping gene test, $B 2 M$ and $G A P D H$ were selected for normalization.

\section{Abbreviations}

BM: bone marrow; BM-MNCs: bone marrow mononuclear cells; BTZM: bortezomib; CLMDZ: calmidazolium chloride; DSS: Durie-Salmon stage; ISS: international scoring system; MGUS: monoclonal gammopathy of undetermined significance; MM: multiple myeloma; MPC: mesangiogenic progenitor cells; $\mathrm{NH}$ : non-hematological; MSCs: mesenchymal stromal cells; PCs: plasma cells; SMM: smoldering multiple myeloma; VEGF: vascular-endothelial growth factor.

\section{Author contributions}

SP and MP conceived and designed the experiments. SP, MM, FM and CS performed the main experiments and analyzed the data. SB, FMP, LM and LN participated in angiogenic sprouting assay. GBP participated in terminal osteogenic differentiation assays. VC performed the gene expression profiling SP, FM, LM, CS and G wrote the manuscript. All authors read and approved the final manuscript.

\section{ACKNOWLEDGMENTS}

Not applicable.

\section{CONFLICTS OF INTEREST}

The authors declare no conflicts of interest

\section{FUNDING}

This work was partially supported by Associazione Italiana contro le leucemie-linfomi e mieloma (A.I.L.)

\section{REFERENCES}

1. Zhou J. The role of the tumor microenvironment in hematological malignancies and implication for therapy. Front Biosci. 2005; 10: 1581-96. https://doi.org/10.2741/1642. [PubMed]

2. Manier S, Sacco A, Leleu X, Ghobrial IM, Roccaro AM. Bone Marrow Microenvironment in Multiple Myeloma Progression. J Biomed Biotechnol. 2012; 2012: 157496. https://doi.org/10.1155/2012/157496. [PubMed]

3. Kawano Y, Moschetta M, Manier S, Glavey S, Görgün GT, Roccaro AM, Anderson KC, Ghobrial IM. Targeting the bone marrow microenvironment in multiple myeloma. Immunol Rev. 2014; 263: 160-72. [PubMed]

4. Basak G, Srivastava A, Malhotra R, Carrier E. Multiple Myeloma Bone Marrow Niche. Curr
Pharm Biotechnol. 2009; 10: 335-46. https://doi. org/10.2174/138920109787847493. [PubMed]

5. Garcia-Gomez A. Multiple myeloma mesenchymal stromal cells: Contribution to myeloma bone disease and therapeutics. World J Stem Cells. 2014; 6: 322-43. [PubMed]

6. McDonald MM, Fairfield H, Falank C, Reagan MR. Adipose Bone, and Myeloma: Contributions from the Microenvironment. Calcif Tissue Int. 2016; 100: 433-48. https://doi.org/10.1007/s00223-016-0162-2. [PubMed]

7. Ribatti D, Vacca A. New Insights in Anti-Angiogenesis in Multiple Myeloma. Int J Mol Sci. 2018; 19: 2031. https:// doi.org/10.3390/ijms19072031. [PubMed]

8. Vacca A, Ria R, Reale A, Ribatti D. Angiogenesis in multiple myeloma. Chem Immunol Allergy. 2014; 99: 18096. https://doi.org/10.1159/000353312. [PubMed]

9. Undale $\mathrm{AH}$, Westendorf JJ, Yaszemski MJ, Khosla S. Mesenchymal Stem Cells for Bone Repair and Metabolic Bone Diseases. Mayo Clin Proc. 2009; 84: 893-902. https://doi.org/10.4065/84.10.893. [PubMed]

10. Kwon HM, Hur SM, Park KY, Kim CK, Kim YM, Kim HS, Shin HC, Won MH, Ha KS, Kwon YG, Lee DH, Kim YM. Multiple paracrine factors secreted by mesenchymal stem cells contribute to angiogenesis. Vascul Pharmacol. 2014; 63: 19-28. https://doi.org/10.1016/j.vph.2014.06.004. [PubMed]

11. Pacini S, Petrini I. Are MSCs angiogenic cells? New insights on human nestin-positive bone marrow-derived multipotent cells. Front Cell Dev Biol. 2014; 2: 20. https:// doi.org/10.3389/fcell.2014.00020.

12. Montali M, Barachini S, Panvini FM, Carnicelli V, Fulceri F, Petrini I, Pacini S. Front Cell Dev Biol. 2016; 4: 114. https://doi.org/10.3389/fcell.2016.00114.

13. Petrini M, Pacini S, Trombi L, Fazzi R, Montali M, Ikehara S, Abraham NG. Identification and Purification of Mesodermal Progenitor Cells From Human Adult Bone Marrow. Stem Cells Dev. 2009; 18: 857-66. https://doi. org $/ 10.1089 / \mathrm{scd} .2008 .0291$. [PubMed]

14. Pacini S, Carnicelli V, Trombi L, Montali M, Fazzi R, Lazzarini E, Giannotti S, Petrini M. Constitutive expression of pluripotency-associated genes in mesodermal progenitor cells (MPCs). PLoS One. 2010; 5: e9861. https://doi. org/10.1371/journal.pone.0009861. [PubMed]

15. Montali M, Panvini FM, Barachini S, Ronca F, Carnicelli V, Mazzoni S, Petrini I, Pacini S. Human adult mesangiogenic progenitor cells reveal an early angiogenic potential, which is lost after mesengenic differentiation. Stem Cell Res Ther. 2017; 8: 106. https://doi.org/10.1186/s13287-017-0562-x. [PubMed]

16. Sunagawa $M$, Kosugi $T$, Nakamura $M$, Sperelakis N. Pharmacological Actions of Calmidazolium, a Calmodulin Antagonist, in Cardiovascular System. Cardiovasc Drug Rev. 2000; 18: 211-21. https://doi. org/10.1111/j.1527-3466.2000.tb00044.x.

17. Fazzi R, Pacini S, Carnicelli V, Trombi L, Montali M, Lazzarini E, Petrini M. Mesodermal progenitor cells (MPCs) differentiate into mesenchymal stromal cells 
(MSCs) by activation of Wnt5/calmodulin signalling pathway. PLoS One. 2011; 6: e25600. https://doi. org/10.1371/journal.pone.0025600. [PubMed]

18. Pacini S, Barachini S, Montali M, Carnicelli V, Fazzi R, Parchi P, Petrini M. Mesangiogenic Progenitor Cells Derived from One Novel CD64(bright)CD31(bright)CD14(neg) Population in Human Adult Bone Marrow. Stem Cells Dev. 2016; 25: 661-73. https://doi.org/10.1089/scd.2015.0344. [PubMed]

19. Roccaro AM, Hideshima T, Raje N, Kumar S, Ishitsuka K, Yasui H, Shiraishi N, Ribatti D, Nico B, Vacca A, Dammacco F, Richardson PG, Anderson KC. Bortezomib mediates antiangiogenesis in multiple myeloma via direct and indirect effects on endothelial cells. Cancer Res. 2006; 66: 184-91. https://doi.org/10.1158/0008-5472.CAN-05-1195. [PubMed]

20. Montali M, Barachini S, Pacini S, Panvini FM, Petrini M. Isolating Mesangiogenic Progenitor Cells (MPCs) from Human Bone Marrow. J Vis Exp. 2016;113. https://doi. org/10.3791/54225. [PubMed]

21. Giuliani N, Morandi F, Tagliaferri S, Lazzaretti M, Bonomini S, Crugnola M, Mancini C, Martella E, Ferrari L, Tabilio A, Rizzoli V. The proteasome inhibitor bortezomib affects osteoblast differentiation in vitro and in vivo in multiple myeloma patients. Blood. 2007; 110: 334-8 https:// doi.org/10.1182/blood-2006-11-059188. [PubMed]

22. Ria R, Solimando A, Melaccio A, Sportelli A, Vacca A. Angiogenesis and Antiangiogenesis in Multiple Myeloma. Mayo Clin Proc. IntechOpen. 2019. https://doi.org/10.5772/ intechopen.76931.

23. Terpos E, Ntanasis-Stathopoulos I, Gavriatopoulou M, Dimopoulos MA. Pathogenesis of bone disease in multiple myeloma: from bench to bedside. Blood Cancer Journal. 2018; 8: 7. https://doi.org/10.1038/s41408-0170037-4. [PubMed]

24. Rajkumar SV. Treatment of Myeloma: Cure vs Control. Mayo Clinic Proc. 2008; 83: 1142-5. https://doi. org/10.4065/83.10.1142. [PubMed]

25. Kyle RA, Gertz MA, Witzig TE, Lust JA, Lacy MQ, Dispenzieri A, Fonseca R, Rajkumar SV, Offord JR, Larson DR, Plevak ME, Therneau TM, Greipp PR. Review of 1027 Patients With Newly Diagnosed Multiple Myeloma. Myeloma. Mayo Clin Proc. 2003; 78: 21-33. https://doi. org/10.4065/78.1.21. [PubMed]

26. Bolzoni M, Donofrio G, Storti P, Guasco D, Toscani D, Lazzaretti M, Bonomini S, Agnelli L, Capocefalo A, Palma BD, Neri A, Nicolini F, Lisignoli G, et al. Myeloma cells inhibit non-canonical wnt co-receptor ror2 expression in human bone marrow osteoprogenitor cells: effect of wnt5a/ ror2 pathway activation on the osteogenic differentiation impairment induced by myeloma cells. Leukemia. 2012; 27: 451-63. https://doi.org/10.1038/leu.2012.190. [PubMed]

27. Kusumbe AP, Ramasamy SK, Adams RH. Coupling of angiogenesis and osteogenesis by a specific vessel subtype in bone. Nature. 2014; 507: 323-8. https://doi.org/10.1038/ nature13145. [PubMed]
28. Wang L, Zhou F, Zhang P, Wang H, Qu Z, Jia P, Yao Z, Shen G, Li G, Zhao G, Li J, Mao Y, Xie Z, et al. Human type $\mathrm{H}$ vessels are a sensitive biomarker of bone mass. Cell Death Dis. 2017; 8: e2760. https://doi.org/10.1038/ cddis.2017.36. [PubMed]

29. Qiang YW, Hu B, Chen Y, Zhong Y, Shi B, Barlogie B, Shaughnessy JD. Bortezomib induces osteoblast differentiation via Wnt-independent activation of -catenin/ TCF signaling. Blood. 2009; 113: 4319-30. https://doi. org/10.1182/blood-2008-08-174300. [PubMed]

30. Landgren O. Monoclonal gammopathy of undetermined significance and smoldering multiple myeloma: biological insights and early treatment strategies. Hematology Am Soc Hematol Educ Program. 2013; 2013: 478-87. https://doi. org/10.1182/asheducation-2013.1.478 [PubMed]

31. Mateos MV1, Hernández MT, Giraldo P, de la Rubia J, de Arriba F, López Corral L, Rosiñol L, Paiva B, Paiva B, Palomera L, Bargay J, Oriol A, Prosper F, Prosper F, López J, Olavarría E, Quintana N, García JL, Bladé J, Lahuerta JJ, San Miguel JF. Lenalidomide plus Dexamethasone for High-Risk Smoldering Multiple Myeloma. N Engl J Med. 2013; 369: 438-47. https://doi. org/10.1056/nejmoa1300439. [PubMed]

32. Sonneveld P, Schmidt-Wolf IG,van der Holt B, Jarari L , Bertsch U, Salwender H, Zweegman S, Vellenga E, Broyl A, Blau IW, Weisel KC, Wittebol S, Bos GMJ, et al. Bortezomib Induction and Maintenance Treatment in Patients With Newly Diagnosed Multiple Myeloma: Results of the Randomized Phase III HOVON-65/ GMMG-HD4 Trial. J Clin Oncol. 2012; 30: 2946-55. https://doi. org/10.1200/jco.2011.39.6820. [PubMed]

33. Costa LJ, Landau H, Venkata JK, Kang Y, Koehne G, Chung DJ, Lendvai N, Bentz T, Giralt SA. Phase 1 Trial of Carfilzomib + Melphalan (CarMel) Conditioning and Autologous Hematopoietic Stem Cell Transplantation (AHSCT) for Relapsed Multiple Myeloma (MM). Biol Blood Marrow Transplant. 2014; 20: S159-S160.. https:// doi.org/10.1016/j.bbmt.2013.12.254.

34. van de Donk NWCJ, Yong K. Oral proteasome inhibitor maintenance for multiple myeloma. The Lancet. 2019; 393: 204-5. https://doi.org/10.1016/S0140-6736(18)33128-3.

35. Morgan G, Dimopoulos M, Gay F, Schjesvold F, Beksac M, Hajek R, Weisel KC, Goldschmidt H, Maisnar V, Moreau P, Min C-K, Pluta A, Chng WJ, et al. Maintenance Therapy with the Oral Proteasome Inhibitor (PI) Ixazomib Significantly Prolongs Progression-Free Survival (PFS) Following Autologous Stem Cell Transplantation (ASCT) in Patients with Newly Diagnosed Multiple Myeloma (NDMM): Phase 3 Tourmaline-MM3 Trial. Biol Blood Marrow Transplant. 2019; 25: S19-S20. https://doi.org/10.1016/j.bbmt.2018.12.087.

36. International Myeloma Working Group. Criteria for the classification of monoclonal gammopathies, multiple myeloma and related disorders: a report of the International Myeloma Working Group. Br J Haematol. 2003; 121: 749-57. [PubMed] 